\title{
The Frequency of Nonmotor Symptoms among Advanced Parkinson Patients May Depend on Instrument Used for Assessment
}

\author{
Nelson Hwynn, ${ }^{1,2}$ Ihtsham U. Haq, ${ }^{3}$ Irene A. Malaty, ${ }^{1}$ Andrew S. Resnick, ${ }^{1}$ Michael S. Okun, ${ }^{1}$ \\ Danica S. Carew, ${ }^{1}$ Genko Oyama, ${ }^{1}$ Yunfeng Dai, ${ }^{4}$ Samuel S. Wu, ${ }^{4}$ Ramon L. Rodriguez, ${ }^{1}$ \\ Charles E. Jacobson IV, ${ }^{1}$ and Hubert H. Fernandez ${ }^{1,4,5}$ \\ ${ }^{1}$ University of Florida Center for Movement Disorders and Neurorestoration, University of Florida, Gainesville, FL 32607, USA \\ ${ }^{2}$ Division of Neurology, Scripps Clinic Torrey Pines, La Jolla, San Diego, CA 92037, USA \\ ${ }^{3}$ Department of Neurology, Wake Forest School of Medicine, Winston-Salem, NC 27157, USA \\ ${ }^{4}$ Department of Biostatistics, University of Florida, Gainesville, FL 32611, USA \\ ${ }^{5}$ Movement Disorders, Cleveland Clinic, Center for Neurological Restoration, Cleveland, OH 44195, USA \\ Correspondence should be addressed to Hubert H. Fernandez, fernanh@ccf.org
}

Received 16 December 2010; Revised 3 March 2011; Accepted 16 May 2011

Academic Editor: Fabrizio Stocchi

Copyright () 2011 Nelson Hwynn et al. This is an open access article distributed under the Creative Commons Attribution License, which permits unrestricted use, distribution, and reproduction in any medium, provided the original work is properly cited.

Background. Nonmotor symptoms (NMS) of Parkinson's disease (PD) may be more debilitating than motor symptoms. The purpose of this study was to determine the frequency and corecognition of NMS among our advanced PD cohort (patients considered for deep brain stimulation (DBS)) and caregivers. Methods. NMS-Questionnaire (NMS-Q), a self-administered screening questionnaire, and NMS Assessment-Scale (NMS-S), a clinician-administered scale, were administered to PD patients and caregivers. Results. We enrolled 33 PD patients (23 males, 10 females) and caregivers. The most frequent NMS among patients using NMS-Q were gastrointestinal (87.9\%), sleep (84.9\%), and urinary (72.7\%), while the most frequent symptoms using NMS-S were sleep $(90.9 \%)$, gastrointestinal $(75.8 \%)$, and mood $(75.8 \%)$. Patient/caregiver scoring correlations for NMS-Q and NMS-S were $0.670(P<0.0001)$ and $0.527(P=0.0016)$, respectively. Conclusion The frequency of NMS among advanced PD patients and correlation between patients and caregivers varied with the instrument used. The overall correlation between patient and caregiver was greater with NMS-Q than NMS-S.

\section{Introduction}

It is becoming increasingly recognized that $\mathrm{PD}$ is a multidimensional disease and that nonmotor symptoms (NMS) can potentially affect quality of life as much or more than motor dysfunction [1]. NMS may be underrecognized by physicians and include such important features as cognitive impairment, depression, and apathy. A recent paper by Carter and colleagues found that NMS may contribute more to caregiver strain and depression than motor symptoms [2]. The NMS Questionnaire (NMS-Q) was devised to screen patients and was recently validated [3]. Subsequently, an NMS Assessment Scale for Parkinson's disease (NMS-S) has been created to assess the frequency and severity of NMS in PD [4].

In a large, recent multicenter study by Barone and colleagues, $98.6 \%$ of PD patients had at least one NMS [5].
However, studies surveying NMS specifically in advanced PD patients have been limited. We suspected that, in this subset of PD patients, NMS may play even greater role and may be more prevalent.

We chose a population with relatively advanced PD, and we aimed in this study to determine the frequency of NMS in PD patients and to evaluate the correlation between PD patient and caregiver perceptions of NMS.

\section{Methods}

Consecutive advanced PD patients with severe motor fluctuations who were candidates undergoing evaluation for either unilateral STN or GPI stimulation were included. All patients signed informed consents to have their data stored in a database in accordance with the Declaration of 
Helsinki. All subjects were between the ages of 18 to 85 , had diagnosis of idiopathic PD confirmed by a movement disorders specialist (UK Brain Bank criteria), and also had a documented greater than 30\% improvement in motor performance following levodopa administration (as measured by the Unified Parkinson's Disease Rating Scale Part III). Subjects were excluded from DBS implantation and consequently from this study if they had significant and active psychiatric disease after a structured clinical interview (SCID) or if demented (DRS score of $<130$ or demented based on neuropsychological profile).

The NMS-S and NMS-Q were separately administered to each subject and caregiver. The NMS-S (clinically driven questionnaire) was administered by a trained member of the movement disorders team just prior to DBS surgery. Patients were separated from their caregivers during scale and questionnaire administration.

The NMS-Q form lists NMS symptoms with a "yes" or "no" format. "Yes" answers are assigned one point; "no" answers are assigned 0 points. The subsets were divided in an attempt to better match comparison to the NMS$S$ as follows: questions 1,3 , and 4-7 were grouped under the gastrointestinal symptoms subset; questions 8-9 were grouped under the urinary symptoms subset; questions 2 , 10-11, and 27-28 were grouped under the miscellaneous symptoms subset; questions $12-13$ and 15 were grouped under the cognition symptoms subset; questions 16-17 were grouped under the mood symptoms subset; questions 18-19 were grouped under the sexual dysfunction symptoms subset; questions 20-21 were grouped under the cardiovascular/falls symptoms subset; questions 22-26 were grouped under the sleep symptoms subset; questions 14, 29-30 were grouped under perceptual symptoms subset.

The NMS-S not only assesses if NMS are present, but the frequency (range of 0-4) and severity (range of 0-3) are also rated. If the product of frequency $\mathrm{x}$ severity is 1 or greater, then 1 point is assigned. The NMS-S was designed for patients to assess their symptoms and not specifically for caregiver assessment. Using the NMS-S form, the subsets were divided as follows: questions 1-2 were grouped under the cardiovascular/falls symptom subset; questions 3-7 were grouped under the sleep/fatigue symptoms subset; questions 8-14 were grouped under the mood symptoms subset; questions 15-17 were grouped under the perceptual symptoms subset; questions 18-20 were grouped under the cognition symptoms subset; questions 21-23 were grouped under the gastrointestinal symptoms subset; questions 24-26 were grouped under the urinary symptoms subset; questions 27-28 were grouped under the sexual dysfunction symptoms subset; questions 29-32 were grouped under the miscellaneous symptoms subset.

Percent prevalence was calculated by form (NMS-Q or NMS-S) and by scorer (patient or caregiver). For each domain and the total score of NMS-Q and NMS$S$, Spearman's rank correlation coefficient was evaluated between patient and caregiver scoring and tested whether the correlation was significantly different from zero.

\section{Results}

Thirty-three PD patients and their caregivers were included in the study. Twenty-six patients eventually underwent STN implantation at our institution. The remaining seven patients subsequently underwent GPi implantation. The average age of PD patients who participated was 61.3 years \pm 8.1 . Twenty three patients were men, and 10 patients were women. The average off-medication UPDRS motor score at baseline was 40.3 points \pm 11.9 .

3.1. NMS-Q. Table 1 summarizes the prevalence and correlation of NMS by patients and caregivers. According to patients, the most common NMS-Q items were GI (87.9\%), sleep $(84.9 \%)$, and urinary $(72.7 \%)$ symptoms. The least prevalent NMS identified by PD patients were perceptual (which included diplopia and delusional thoughts) (18.2\%), mood (48.5\%), cardiovascular/falls $(54.6 \%)$ and sexual (54.6\%) symptoms.

According to caregivers using the NMS-Q, the most prevalent NMS symptoms were sleep (90.9\%), GI (81.8\%), and miscellaneous (which included unexplained pain, anosmia, weight loss, and excessive sweating) (69.7\%) items. The least prevalent NMS caregivers identified were perceptual (15.2\%), cardiovascular (42.4\%), and mood (54.6\%) symptoms.

3.2. NMS-S. Table 1 summarizes the prevalence and correlation of NMS by patients and caregivers. According to patients, the most common NMS using the NMS-S were sleep (90.9\%), GI (75.8\%), and $\operatorname{mood}(75.8 \%)$ symptoms. The least prevalent NMS in PD patients were perceptual $(18.2 \%)$, cardiovascular $(45.5 \%)$, and sexual (45.5\%) symptoms.

Using the NMS-S, caregivers identified sleep (93.9\%), GI (72.7\%), and mood (72.7\%) and cognition (69.7\%) symptoms most commonly. The least prevalent NMS that their caregivers identified was perceptual (15.2\%), cardiovascular (33.3\%), and urinary (48.5\%) symptoms.

3.3. Patient and Caregiver Correlations. The highest correlation of identified NMS symptoms using NMS-Q between patients and their caregivers was with mood (0.65), sleep (0.62), urinary (0.62), and miscellaneous (0.54) symptoms. The lowest correlation of identified NMS symptoms using NMS-Q between patients and their caregivers was with cardiovascular (0.36), sexual (0.42), perceptual (0.46), and gastrointestinal (0.53) symptoms (Table 1 ).

The highest correlation of identified NMS symptoms using NMS-S between patients and their caregivers was with cognition (0.62), mood (0.55), and sexual symptoms (0.50). The lowest correlation of identified NMS symptoms using NMS-S between patients and their caregivers was with miscellaneous (0.38), cardiovascular (0.39), and perceptual subsets (0.40) (Table 1).

Spearman's correlation coefficient of NMS-Q scores between patient and caregiver was $0.670(P$ value $<0.0001)$. Spearman's correlation coefficient of NMS-S was 0.527 $(P$ value $=0.0016)$. 
TABLe 1: Frequency of nonmotor symptoms. In the NMS-Q, at least 1 question in the category is "yes." In the NMS-S, Severity "Frequency" is greater than 0 in at least 1 item in the subgroups.

\begin{tabular}{|c|c|c|c|c|}
\hline NMS-Q & $\begin{array}{l}\text { Patient evaluation } \\
\text { Percent prevalence }\end{array}$ & $\begin{array}{l}\text { Caregiver evaluation } \\
\text { Percent prevalence }\end{array}$ & Correlation & P value \\
\hline Gastrointestinal & $87.9 \%$ & $81.8 \%$ & 0.53 & 0.002 \\
\hline Sleep & $84.9 \%$ & $90.9 \%$ & 0.62 & 0.0001 \\
\hline Urinary & $72.7 \%$ & $63.6 \%$ & 0.62 & 0.0001 \\
\hline Miscellaneous* & $69.7 \%$ & $69.7 \%$ & 0.54 & 0.001 \\
\hline Cognition & $63.6 \%$ & $66.7 \%$ & 0.46 & 0.007 \\
\hline Sexual & $54.6 \%$ & $57.1 \%$ & 0.42 & 0.03 \\
\hline Cardiovascular/falls & $54.6 \%$ & $42.4 \%$ & 0.36 & 0.04 \\
\hline Mood & $48.5 \%$ & $54.6 \%$ & 0.65 & $0<.0001$ \\
\hline Perceptual $^{* *}$ & $18.2 \%$ & $15.2 \%$ & 0.46 & 0.007 \\
\hline NMS-S & $\begin{array}{l}\text { Patient evaluation } \\
\text { Percent prevalence }\end{array}$ & $\begin{array}{l}\text { Caregiver evaluation } \\
\text { Percent prevalence }\end{array}$ & Correlation & P value \\
\hline Sleep/Fatigue & $90.9 \%$ & $93.9 \%$ & 0.46 & 0.01 \\
\hline Gastrointestinal & $75.8 \%$ & $72.7 \%$ & 0.48 & 0.005 \\
\hline Mood & $75.8 \%$ & $72.7 \%$ & 0.55 & 0.0009 \\
\hline Miscellaneous*** & $72.7 \%$ & $66.7 \%$ & 0.38 & 0.03 \\
\hline Cognition & $60.6 \%$ & $69.7 \%$ & 0.62 & 0.0001 \\
\hline Urinary & $57.6 \%$ & $48.5 \%$ & 0.47 & 0.01 \\
\hline Sexual & $45.5 \%$ & $53.6 \%$ & 0.50 & 0.01 \\
\hline Cardiovascular/falls & $45.5 \%$ & $33.3 \%$ & 0.39 & 0.02 \\
\hline Perceptual**** & $18.2 \%$ & $15.2 \%$ & 0.40 & 0.02 \\
\hline
\end{tabular}

\section{Discussion}

The most frequent NMS in Chaudhuri's tested cohort of PD patients using the NMS-Q included nocturia (66.7\%), urinary urgency $(61 \%)$, constipation $(46.7 \%)$, memory (43.9\%), and sadness (44.7\%) [3]. Further, in the Chaudhuri and Martinez-Martin cohort, the most prevalent symptoms using the NMS-S included nocturia $(59.5 \%)$, urinary urgency (53.6\%), constipation (50.2\%), depression (48.2\%), insomnia (44.3\%), and concentration (44\%) [4]. A comparison to our cohort of advanced (presurgical) PD patients reveals similar findings but different rank order. When using the NMS-Q, patients in our study reported gastrointestinal, sleep, and urinary symptoms, and when using the NMSS, PD patients reported sleep, gastrointestinal, and mood symptoms most frequently. The reasons for the differences include different cohorts, different disease severities, and a different demographic population. Also this advanced PD patient population was screened for cognitive findings and excluded for severe mood disorders prior to DBS surgery, and this may have also accounted for some of the differences.

The frequency of NMS among advanced PD patients varied in our study depending on the type of instrument utilized. For example, the frequency of mood symptoms was among the top 3 in the NMS-S, but in the bottom 3 in the NMS-Q. This could be a reflection of the difference of emphasis between the scales. In the NMS-Q, there were 2 questions that made up the mood subset, while, in the NMS-S there were 7. Devoting more questions to a particular symptom may increase sensitivity of the instrument for identifying milder manifestations of difficulty. In another example, in the NMS-Q scale, the gastrointestinal subset was composed of 9 questions, while in the NMS-S scale, the gastrointestinal subset was composed of only 3 questions. Finally, another important difference is that NMS-Q is patient driven while the NMS-S is clinician driven. In the mood example, clinician interpretation of a patient's answer may impact reporting differently than self-reported information. Self-report for items such as sexual difficulties may be more likely to capture full disclosure than faceto-face reporting, within a clinician-administered scale. For these reasons, the NMS frequencies differ and are instrument driven if they are compared head to head as a screening tool. This methodology of this study simplifies the results of the NMS-S, which is designed to assess impact of the NMS rather than simply screening for its presence as in the NMS-Q.

The correlation of NMS between patients and caregivers was also variable depending on the instrument and the symptom subset. In general, there was higher overall correlation in identifying NMS between PD patients and 
caregivers using the NMS-Q than with NMS-S. The NMS$Q$ had 5 subsets where the correlation between patient and caregiver responses was greater than 0.50 , while the NMS-S had only 3 reaching this level. The 3 subsets with the highest patient/caregiver correlation using NMS-Q were mood, sleep, and urinary symptoms, and this differed from the 3 subsets with the highest patient/caregiver correlation using NMS-S where cognition, mood, and sexual items emerged. Of these most correlated NMS, only mood was within the highest correlated symptoms when using both instruments. The lack of correlation using the other symptom subsets between the patients and their caregivers may have been due to the caregiver's ability to recognize but not accurately rank the severity of symptoms close to how patients would report them. The NMS-Q is useful to screen for the presence of nonmotor symptoms in Parkinson's patients but does not reveal the extent that these symptoms affect quality of life. The NMS-S on the other hand takes into consideration the factors of duration and severity of symptoms that are present and extent that it affects the quality of life, but the instrument is more complicated and correct completion may require a trained member of the clinic/research team and not by patients themselves. The NMS-S was not designed to be administered to caregivers, as they are less apt than patients to more fully understand both the severity and duration of NMS that patients experience, which explains why there is lower correlation between patient and caregiver scores using the NMS-S than NMS-Q.

There were several limitations to our study. The small sample size was the greatest limitation. Furthermore, the prevalence of mood symptoms in this cohort may not have been truly representative of the general advanced PD state since unstable psychiatric illness and neuropsychological issues were screened out as part of establishing DBS candidacy. The data do speak to how commonly NMS occurs and that there may be issues with clinicians recognizing these features especially when focusing on motor issues and, interestingly, may be variably identified depending on which scale or questionnaire they use. Clinicians providing care and especially those providing DBS should be aware of the instrument-dependent nature of their recognition of these issues. The NMS-Q had better correlation between patients' and caregivers' reported symptoms, while the NMS-S offers the additional dimension of addressing severity of symptoms that are identified.

In summary, while these 2 instruments have their weaknesses and differences in instrument properties, they have been used in previous epidemiological studies as a basis for determining frequencies. We realize that while our $\mathrm{N}$ is small and the population is indeed biased to the "surgical patient." this may prevent us from determining the true prevalence of NMS in advanced PD and explains the difference in results between our cohort and prior results reported by Chaudhuri. Our emphasis was not as much on the accurate accounting of each NMS, but more that, when converting these scale responses to a simple "symptom is present" versus "symptom is absent," the responses indeed varied even if the two instruments were administered by the same individuals (patient and caregivers); literally, one instrument right after the other.

\section{References}

[1] W. Poewe, "Clinical measures of progression in Parkinson's disease," Movement Disorders, vol. 24, no. 2, supplement, pp. S671-S676, 2009.

[2] J. H. Carter, B. J. Stewart, K. S. Lyons, and P. G. Archbold, "Do motor and nonmotor symptoms in PD patients predict caregiver strain and depression?" Movement Disorders, vol. 23, no. 9, pp. 1211-1216, 2008.

[3] K. R. Chaudhuri, P. Martinez-Martin, A. H. V. Schapira et al., "International multicenter pilot study of the first comprehensive self-completed nonmotor symptoms questionnaire for Parkinson's disease: the NMSQuest study," Movement Disorders, vol. 21, no. 7, pp. 916-923, 2006.

[4] K. R. Chaudhuri and P. Martinez-Martin, "Quantitation of non-motor symptoms in Parkinson's disease," European Journal of Neurology, vol. 15, no. 2, supplement, pp. 2-8, 2008.

[5] P. Barone, A. Antonini, C. Colosimo et al., "The PRIAMO study: a multicenter assessment of nonmotor symptoms and their impact on quality of life in Parkinson's disease," Movement Disorders, vol. 24, no. 11, pp. 1641-1649, 2009. 


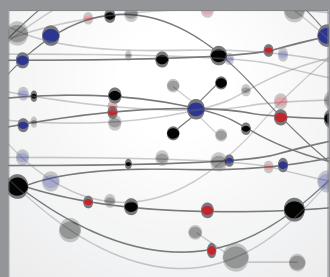

The Scientific World Journal
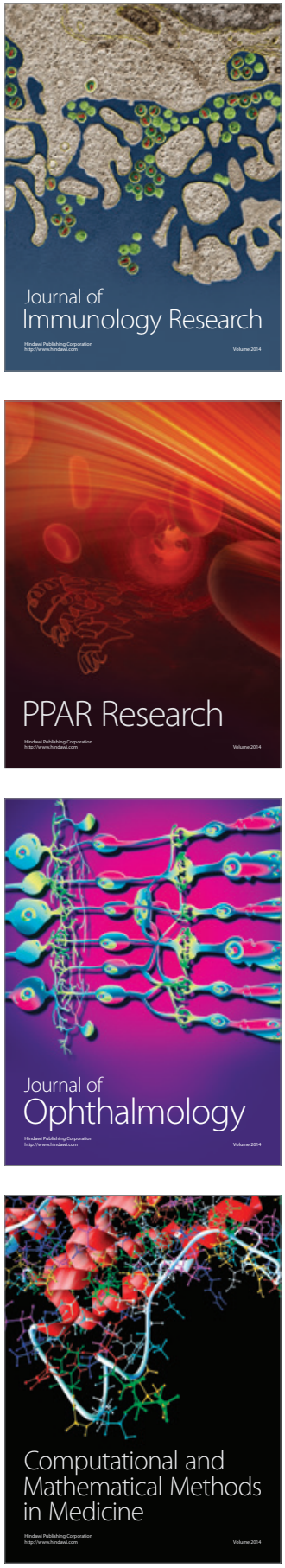

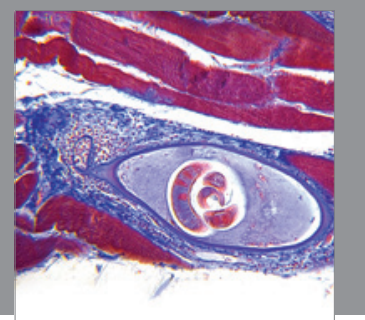

Gastroenterology

Research and Practice
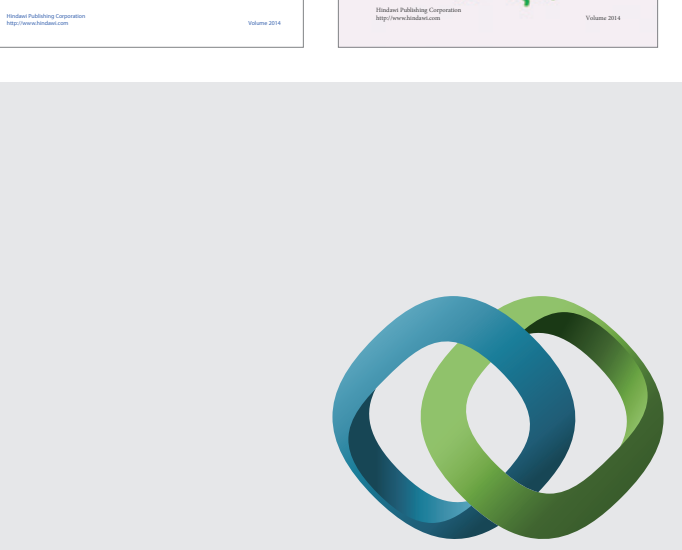

\section{Hindawi}

Submit your manuscripts at

http://www.hindawi.com
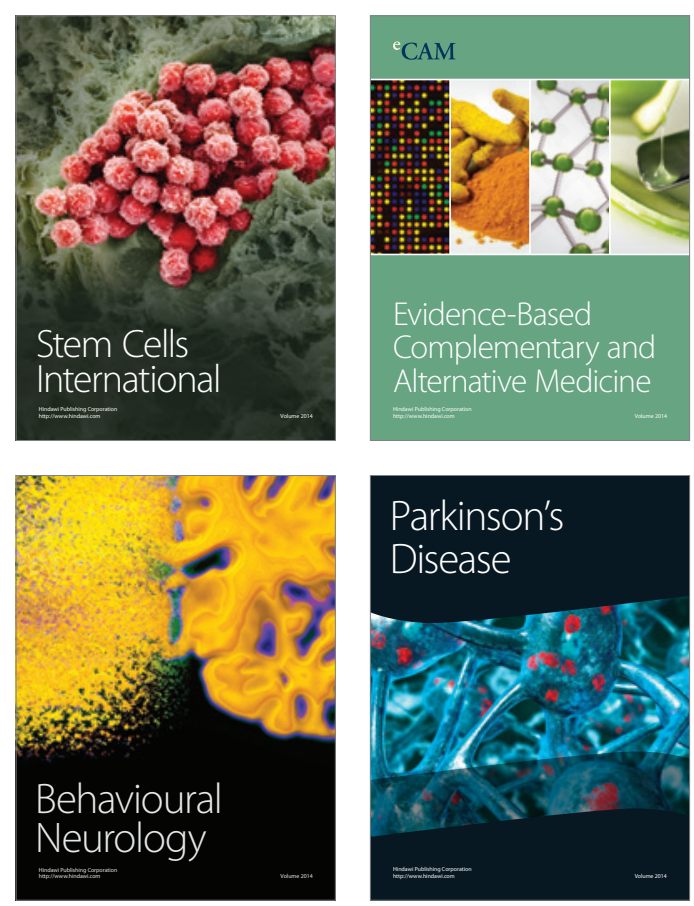

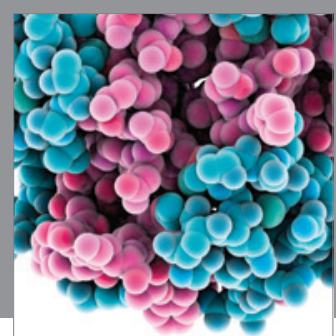

Journal of
Diabetes Research

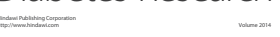

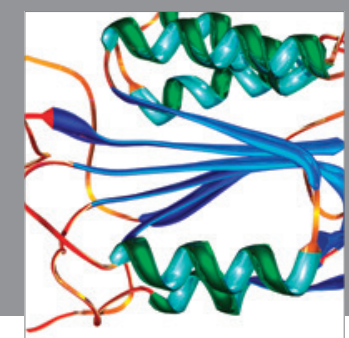

Disease Markers
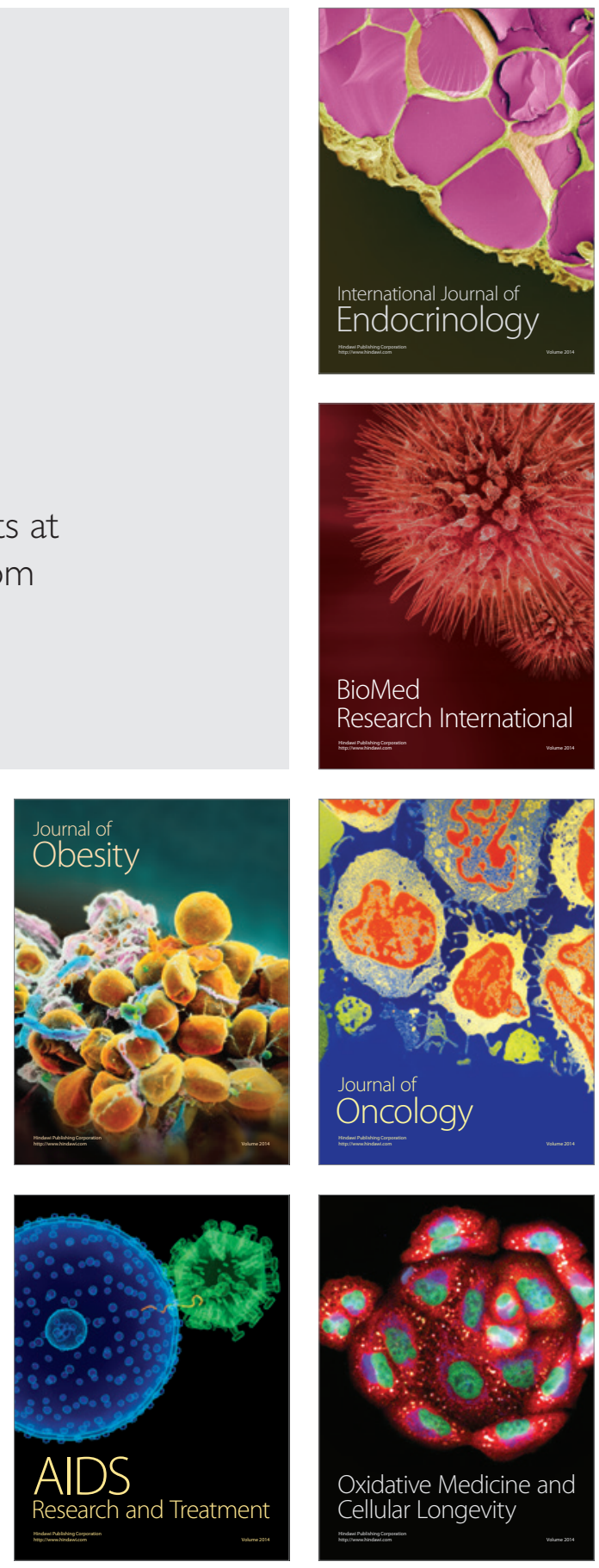